D.O.I.: $10.3895 / \mathrm{S} 1808-04482013000400010$

\title{
DIFUSÃO DA ISO 14001 NO BRASIL: ANÁLISE COMPARATIVA DOS ESTADOS E DOS SETORES DE ATUAÇÃO
}

\section{DISSEMINATION OF ISO 14001 IN BRAZIL: COMPARATIVE ANALYSIS OF STATES AND SECTORS OF ACTIVITY}

\author{
Leonardo de Lima Moura ${ }^{1}$; Ronaldo Ferreira da Silva ${ }^{2}$ \\ ${ }^{1}$ Instituto Brasileiro de Mercado de Capitais - IBMEC - Rio de Janeiro - Brasil \\ mouraventura@uol.com.br \\ ${ }^{2}$ Universidade Federal Fluminense - UFF - Rio de Janeiro - Brasil \\ ronaldorubanouff@gmail.com
}

\begin{abstract}
Resumo
A intensificação da preocupação ambiental por parte do governo e dos stakeholders tem feito com que a adoção do sistema de gerenciamento ambiental pelas organizações venha aumentando consideravelmente nos últimos anos. A inserção de sistemas de gerenciamento tem sido realizada com base em normativas certificadoras como a ISO 14001 atualmente associada a reconhecimento e grande popularidade entre as empresas. Como ainda há uma carência de estudos analisando a temática ISO 14001 no Brasil, o presente estudo visou analisar a evolução da norma de certificação ISO 14001:2004 no período de janeiro de 2007 a dezembro de 2012 com base nas informações presentes no site do Inmetro. Como resultados da pesquisa obteve-se que o estado de São Paulo é o estado com o maior número de certificações válidas e emitidas e que os setores de atuação Atividades Imobiliárias, locações e prestação de serviços e Transporte; armazenagens e telecomunicação apresentam grande parte das certificações válidas no período de 2007 a 2012, certificações estas emitidas em sua grande maioria pelo organismo certificador BRTÜV Avaliações da Qualidade Ltda.
\end{abstract}

Palavras-chave: ISO 14001; sistema de gestão ambiental; setores de atuação; estados.

\section{Introdução}

A intensificação da preocupação ambiental por parte do governo e dos stakeholders tem feito com que a adoção do sistema de gerenciamento ambiental pelas organizações venha aumentando consideravelmente. Além das preocupações ambientais, existem outros fatores estimuladores da adoção deste sistema pelas as empresas, dentre os quais pode se citar: os benefícios oriundos uma vez que as empresas passam a ter uma vantagem competitiva frente às demais e a redução dos custos oriundos dos processos produtivos ( JABBOUR et al, 2012).

No passado, a regulamentação ambiental apresentava um caráter impositivo baseado em regulamentos obrigatórios, como por exemplo, o estabelecimento de limites de emissões de 
poluentes. Apesar de esta política ter contribuído significativamente para a redução de poluição industrial também se mostrou inflexível e dispendiosa para os processos produtivos. A utilização de incentivos econômicos por meio de impostos e licenças negociaveis se apresentou como uma alternativa, no entanto a projeção da eficiência desses instrumentos para diversos poluentes se mostrou extremamente complexa (ZIEGLER; NOGAREDA, 2009).

Em virtude disso, a evolução deste sistema de caratér impositivo para um sistema de gerenciamento ambiental voluntário tem sido incentivada pelo mercado internacional, mudanças regulatórias, demanda por gestão da qualidade e a propria conscientização pública. As negociações da Organização Mundial do Comércio (OMC) em 1986 que buscaram minimizar as barreiras nãotarifárias e a Rio- 92 que enfatizou a necessidade do compromisso ambiental para atender os principios da sustentabilidade podem ser considerados marcos para o desenvolvimento de uma política direcionada para o sistema de gerenciamento ambiental voluntário (MASSOUD et al, 2010).

O conceito sistema de gerenciamento ambiental voluntário pode ser definido como uma alternativa atualmente utilizada por empresas no intuito de melhorar a qualidade dos seus processos produtivos do ponto de vista ambiental através do controle e da redução da poluição do meio ambiente. O resultado associado a adoção deste sistema é a maior proatividade frente a questão ambiental, uma vez que há a modernização das atividades realizadas reduzindo assim o número de impactos ambientais e a emissão de multas pelos organismos de controle (PRAKASH; POTOSKI, 2012).

A introdução do sistema de gerenciamento ambiental tem sido realizada com base em normas certificadoras. Normativas estas que originalmente surgiram no inicio do século XX com o intuito de reduzir a variabilidade dos processos e produtos, promovendo assim a padronização do processo produtivo e consequentemente o comércio internacional pela eliminação dos obstáculos associados as divergências de fabricação dos países (MARIMON et al, 2010).

Em 1996, houve a introdução pela International Organization for Standardization da norma certificadora que mais tem sido analisada por pesquisas ultimamente a ISO 14001 que estabelece os procedimentos voluntários que uma empresa precisa adotar para a implementação de um sistema de gerenciamento ambiental (GRAVONSKI et al, 2008).

A quantidade considerável de pesquisas associadas a ISO 14001 se deve ao número crescente de organizações certificadas, certificação esta atualmente associada a reconhecimento e grande popularidade entre as empresas. Em nível mundial, estudos tem buscado analisar a influência da certificação ISO 14001em fatores econômicos, de mercado e regulatórios, sendo que alguns correlacionaram a certificação com o aumento dos lucros, investimentos de capital estrangeiro e aumento da exportação (QI et al, 2011). 
No entanto conclusões acerca da efetividade da ISO 14001 sobre a melhoria da gestão ambiental empresarial ainda são divergentes. Por um lado, alguns estudos demonstram que a certificação pela ISO 14001 pode reduzir os impactos ambientais associados ao processo produtivo e aumentar a proatividade dessas empresas frente às legislações vigentes. Enquanto outros estudos encontraram poucas evidências de melhoria da gestão ambiental além de destacarem os altos custos associados a implementação desta certificação (ARIMURA et al, 2011).

Em relação ao Brasil, num estudo pioneiro Pombo e Magrini (2008) analisaram o estado de certificação em conformidade com a norma ISO 14001:2004 no país no periodo de maio/junho de 2005 a maio/junho de 2006 e obtiveram que naquele momento havia 2300 certificações o que demonstrou que o Brasil apresentava uma postura proativa das empresas brasileiras frente às exigências do mercado. Dentre os setores os que apresentavam maior destaque eram: automotivo, petroquímico e químico e o setor de prestação de serviços.

Oliveira et al (2010) num foco mais direcionado para o estado de São Paulo, analisou que em 2008 o Brasil apresentava 2447 certificações, sendo que destas 26,65\% eram provenientes do Estado de São Paulo. No entanto, ressalta que este número levava em consideração as certificações baseadas em versões antigas da norma e certificações diferentes para processos produtivos de uma mesma organização.

Como ainda há uma carência de estudos analisando a temática ISO 14001 no Brasil, o presente estudo visa analisar a evolução da norma de certificação ISO 14001:2004 no período de janeiro de 2007 a dezembro de 2012 com base nas informações presentes no site do Inmetro.

Para a fundamentação do objeto de pesquisa do estudo primeiramente foi realizada uma revisão da literatura acerca da gestão ambiental, sistema de gestão ambiental e ISO 14001.

\section{Revisão bibliográfica}

\subsection{Gestão ambiental}

A gestão ambiental pode ser definida como um conjunto de procedimentos adotados por uma organização para gerenciar os seus processos produtivos levando em consideração os impactos ambientais associados a estas atividades, observando também as expectativas dos diferentes stakeholders frente à postura adotada pela empresa (LEÃO et al, 2012).

A variável ambiental tem se tornado cada vez mais importante nos processos produtivos em função do aumento da conscientização do consumidor e do crescente interesse na forma como os produtos e serviços são produzidos, fazendo com que as empresas necessitem implementar processos mais limpos para produção de bens e serviços (PEIXE et al, 2013).

O aumento da conscientização ambiental por parte da população é impulsionado principalmente pela deterioração crescente do meio ambiente, como por exemplo, a diminuição de 
matérias-primas, excesso de resíduos, aumento dos níveis de poluição e do aquecimento global, deterioração esta que é consequência dos processos produtivos atualmente presentes nas organizações (PRAJOGO et al, 2012).

Heras-Saizarbitoria et al (2011) relatam que a inserção da variável ambiental em processos produtivos tem sido analisada por diferentes óticas pelos autores. Alguns autores (HART, 1995; PORTER e VAN DER LINDE,1995; SHRIVASTAVA, 1995; TRUNG e KUMAR, 2005) destacam que a gestão ambiental pode ser considerada uma ferramenta que permite as organizações melhorarem os níveis de desempenho e obter vantagem competitiva frente as demais. Outros autores (JAFFE et al, 1995; WALLEY e WHITEHEAD, 1994), ressaltam que as maiores consequencias relacionadas a inserção de práticas ambientais na produção são altos custos de implementação e pequenos benefícios.

Apesar da variabilidade de visões acerca da gestão ambiental, um dos principais direcionadores da forma como a gestão ambiental será considerada no planejamento estratégico das empresas é o estágio ambiental evolutivo da empresa, que segundo Jabbor et al (2012) pode ser definido em:

- Reativo: Neste estágio evolutivo, a organização busca apenas atender a legislação ambiental vigente, o foco do sistema de gestão ambiental é evitar a ocorrência de problemas ambientais e a empresa não se envolve em atividades externas relacionadas ao meio ambiente. Não há um setor específico para a gestão ambiental e há pouca autoridade dos responsáveis pela gestão ambiental na estrutura organizacional.

- Preventivo: Neste estágio, a organização apresenta um planejamento estratégico voltado para otimização do uso de recursos naturais pela inserção de práticas de ecoeficiência e aplicação de principios ambientais como os 3Rs ( reduzir, reutilizar e reciclar). As questões referentes a gestão ambiental passam a ser discutidas pelas diversas áreas da organização e algumas atividades externas referentes ao meio ambiente passam a ser realizadas.

- Proativo: É o último estágio evolutivo ambiental e neste estágio a variável ambiental se torna elemento central no planejamento estratégico das empresas e na criação de vantagem competitiva. É verificado que a área responsável pela gestão ambiental é ativa e suas ações são integradas a outras áreas da organização. As empresas começam a adotar inovações tecnológicas, principalmente no desenvolvimento de produtos com baixo impacto ambiental.

Independentemente do estágio evolutivo em que as organizações se encontram, qualquer procedimento de inserção da variável ambiental em processos produtivos dependerá da adoção de um sistema de gerenciamento ambiental que possa atender os objetivos ambientais da empresa frente as necessidades dos stakeholders. 


\subsection{Sistema de gerenciamento ambiental}

Um sistema de gerenciamento ambiental (SGA) é composto por um conjunto de políticas internas, planos e ações de implementação voltado para a organização como um todo e considerando as relações da mesma com o meio ambiente. Embora haja uma variabilidade entre as características específicas do SGA, todos buscam de uma forma geral os seguintes objetivos: criação de metas quantificáveis para a redução dos impactos ambientais, verificação do progresso da implementação e correção dos desvios encontrados (DARNALL et al, 2008).

Apenas a adoção de um SGA não garante melhorias no desempenho ambiental de uma organização. No intuito de se tornar uma ferramenta eficaz, é indispensável que uma nova visão estratégica permeie todos os níveis hierárquicos da organização desde a alta gerência até o operacional. Esta nova visão deve ser capaz de incluir no seu processo de sistema de gestão ambiental a ferramenta PDCA de Deming ( Planejar- Realizar- Checar- Agir) (IRALDO et al, 2009).

Um dos principais objetivos do desenvolvimento desta nova visão é criar um clima organizacional propício para a redução das possíveis resistências internas, a níveis aceitáveis. A adoção de um SGA pode implicar em mudanças significativas na gestão e na cultura organizacionais, tornando indispensável que a implantação seja analisada como uma oportunidade de mudança e melhorias (CAMPOS et al, 2013).

$\mathrm{Na}$ literatura, um dos pilares considerados importantes para a inserção de um sistema de gestão ambiental é o recursos humanos, relacionando a relevância das práticas da gestão de pessoas para a melhoria da gestão ambiental. Jabbour et al ( 2009) acrescenta que os gestores que desejam inserir e aperfeiçoar seus sistemas de gestão ambiental devem promover a inserção da dimensão ambiental nos recursos humanos.

A multiplicidade dos elementos associados a implementação e manutenção de sistemas de gestão ambiental demonstram a importância de enfoques sistêmicos capazes de abordar a totalidade e a complexidade da questão ambiental em relação as atividades realizadas. Para as empresas, o enfoque sistêmico permite analisar o meio ambiente de forma holística e definir cenários a longo prazo, considerando-se os objetivos das instituições (SEIFFERT, 2011).

Uma das ferramentas que tem sido mais utilizada para a adoção de enfoques sistêmicos em relação a variável ambiental em processos produtivos são as normas certificadoras voluntárias como a ISO 14001 que tem se configurado como uma ferramenta direcionadora para a implementação de um SGA que atenda as necessidades da organização e dos stakeholders envolvidos. 


\subsection{Norma de certificação ambiental voluntária}

A partir da segunda metade da década de 90, muitas empresas passaram a considerar a gestão ambiental uma prioridade corporativa em resposta a preocupação crescente dos stakeholders em relação aos impactos ambientais associados aos processos produtivos. Em virtude disso, houve um aumento considerável de certificações pela norma ISO 14001, normativa esta de caráter internacional que busca padronizar a implementação de sistemas de gestão ambiental por organizações (CAÑÓN- DE- FRANCIA; GARCÉS-AYERBE, 2009).

No entanto, para que a empresa receba a certificação ISO 14001, é necessário que haja uma prévia revisão completa das práticas ambientais e sistemas adotados, a formulação e implementação de um plano de ação para a gestão ambiental, a identificação da governança interna e um plano de correção dos problemas ambientais. Requisitos estes que, devido ao estágio evolutivo ambiental em que a empresa se encontra, muitas vezes configuram-se como barreiras iniciais da inserção de um sistema de gestão ambiental certificado pela ISO 14001 (ZIEGLER, NOGAREDA; 2009).

Em relação aos requisitos estabelecidos pela ISO 14001, no escopo da sua descrição nota-se que as instruções necessárias para a execução de um SGA são de caráter genérico. Não há instruções especificas sobre com as rotinas devem ser formuladas, implementadas ou gerenciadas em virtude disso a interpretação e adaptação da normativa a realidade da empresa são etapas importantes para o processo de certificação (OLIVEIRA et al, 2010).

Para o atendimento destas necessidades, as empresas que buscam certificar seus sistemas de gerenciamento ambiental pela ISO 14001 incorrem em custos elevados dentre os quais podem se destacar os custos relacionados à criação de um novo sistema de gerenciamento ambiental ou modificação do existente. Para o atendimento dos pré-requisitos da ISO 14001 há a necessidade de gastos com equipamentos, documentação, treinamentos e consultores ambientais, gastos estes que combinados oneram em média a empresa em média US \$ 239 a \$1,37 por empregado,dependendo dos recursos internos da organização (DARNALL, 2006).

Apesar dos custos envolvidos, estudos relatam que a adoção da ISO 14001 pode servir como um dispositivo legitimador das práticas ambientais adotadas pela organização, informando as partes interessadas que há uma postura proativa da empresa frente ao meio ambiente. Além disso, pode se configurar como uma ferramenta de redução de custos envolvidos no processo produtivo por meio da redução dos recursos utilizados (POTOSKI; PRAKASH, 2005).

As divergências apresentadas pela literatura em relação aos benefícios associados a implantação de certificações ambientais pela ISO 14001, demonstra a importância da realização de mais pesquisas de carater quali-quantitativo que busquem analisar o processo de adoção da ISO 14001 pelas empresa de diversos países. 


\section{Metodologia}

Esta pesquisa é classificada, segundo seus objetivos mais gerais, como uma pesquisa de caráter exploratório, já que busca como descrito por Gil (2010) proporcionar maior familiaridade dos pesquisadores em relação a um tema, tema este que neste caso é o processo de certificação pela ISO 14001 no Brasil. O caráter exploratório da pesquisa, como tem o intuito de tornar um problema mais explícito para a futura construção de hipóteses, faz com que o planejamento desta pesquisa seja mais flexível, pois se busca considerar os aspectos mais relativos em relação ao fato em destaque. Gil (2010) destaca que a coleta de dados pode ocorrer de diversas formas, sendo que nesta pesquisa foi adotado o modelo mais usual descrito pelo mesmo com algumas adaptações inerentes da pesquisa: levantamento bibliográfico, levantamento de dados e análise dos dados coletados.

Em relação ao levantamento bibliográfico buscou-se por meio deste contextualizar a questão apresentada como motivadora da pesquisa frente a outros estudos estabelecidos na literatura que abordam esta temática.

Antes da realização do processo de levantamento de dados, estabeleceu-se a delimitação do problema, o que corrobora Gil (2010) que ressalta que o problema quando apresentado tende a ser formulado em caráter muito amplo, requerendo por isso algum tipo de delimitação. Em relação à problemática envolvendo a certificação pela ISO 14001 no Brasil, delimitou-se a análise do processo de certificação para o período de janeiro de 2007 a dezembro de 2012, o intuito desta delimitação foi analisar o processo de certificações no Brasil nos últimos cinco anos.

No presente trabalho, o levantamento e a análise dos dados referentes ao processo de certificação pela ISO 14001 no Brasil foram realizados tendo como fonte de pesquisas principais o Inmetro, órgão responsável pelo credenciamento de organismos certificadores da ISO 14001.

O levantamento de dados apresenta segundo Gil (2010) entre as principais vantagens: conhecimento direto da realidade, economia e rapidez e a quantificação. Quantificação esta que na presente pesquisa foi referente aos seguintes fatores: número de certificações emitidas por estado, número de certificações válidas por estado, número de certificações válidas por código NACE e número de certificações emitidas por organismos credenciados pelo Inmetro.

$\mathrm{Na}$ análise de dados, os pesquisadores, seguindo recomendação de Gil (2010), optaram pela elaboração de instrumentos analiticos para organizar e relacionar os dados. Os dados coletados então foram submetidos a prévio tratamento estatistico e a posterior apresentação dos dados em figuras e tabelas.

A pesquisa apresenta limitações uma vez que as unidades de negócio não necessitam informar ao Inmetro sobre a certificação ISO 14001 inserida no seu processo produtivo. 


\section{Resultados e discussão}

\subsection{Certificações emitidas por Estado}

Após a determinação do número de certificações emitidas por ano, detalhou-se o percentual de certificações emitidas por estado no período analisado e os resultados são apresentados na Tabela 1 :

Tabela 1- Percentual de certificações emitidas por Estado

\begin{tabular}{|c|c|}
\hline Estados da Federação & Percentual das certificações emitidas \\
\hline São Paulo & $49,2 \%$ \\
\hline Paraná & $16,3 \%$ \\
\hline Santa Catarina & $7,9 \%$ \\
\hline Rio de Janeiro & $4,6 \%$ \\
\hline Minas Gerais & $3,8 \%$ \\
\hline
\end{tabular}

Fonte: Os autores

Dentre os estados com o maior número de certificações emitidas no período, observa-se que São Paulo é o estado com o maior número de unidades de negócio certificadas, apresentando 49,2\% das certificações totais emitidas pelos organismos certificados pelo Inmetro, resultado este que corrobora o trabalho de Pombo e Magrini (2008) que determinou que São Paulo no período estudado era o estado com o maior número de certificações.

Em relação aos demais estados, nota-se que o segundo estado é o Paraná com cerca de metade do percentual de certificações emitidas em comparação ao estado de São Paulo. Junto os dois estados apresentam $65,5 \%$ das certificações emitidas no período analisado. O terceiro estado é Santa Catarina com menos da metade do percentual de certificações emitidas em relação ao Estado de Paraná, seguido pelos estados de Rio de Janeiro e Minas Gerais.

Em relação aos resultados encontrados observa-se um predomínio dos estados Sudeste e Sul no percentual de certificações emitidas, o que corrobora Pombo e Magrini (2008), embora neste estudo os estados do Rio de Janeiro e Minas Gerais estivessem respectivamente na segunda e terceira posições no percentual de certificações emitidas e que as posições referentes à Paraná e Santa Catarina fossem respectivamente a quinta e sexta. Nota-se então um aumento no número de certificações no período estudado em relação aos demais estados do Sudeste.

\subsection{Certificações válidas por Estado}

Após a avaliação do percentual de certificações emitidas por estado, analisou-se o percentual de certificações válidas por estado e tal resultado encontra-se expresso na Tabela 2:

Tabela 2- Percentual de certificações válidas por Estado

\begin{tabular}{|c|c|}
\hline Estado & Percentual de certificações válidas (\%) \\
\hline São Paulo & 37,2 \\
\hline Paraná & 16,7 \\
\hline Santa Catarina & 13,2 \\
\hline Rio de Janeiro & 6,3 \\
\hline Bahia & 4,0 \\
\hline
\end{tabular}

Fonte: Os autores 
Entre as certificações válidas, nota-se que São Paulo apresenta o maior percentual com $37,2 \%$ das certificações válidas no território brasileiro no período analisado. Nas três respectivas posições seguintes, observa-se uma manutenção dos estados que apresentavam o maior número de certificações emitidas, Paraná, Santa Catarina e Rio de Janeiro.

A tabela demonstra uma proximidade entre as certificações válidas em Santa Catarina e Paraná com uma diferença de 3,5\%. Já em relação ao Rio de Janeiro, analisa-se que este estado apresenta menos da metade do percentual de certificações válidas em comparação com Santa Catarina. Em comparação ao número de certificações emitidas, observa-se uma alteração na quinta posição com a presença do estado da Bahia com 4,0\% das certificações válidas no lugar de Minas Gerais que apresentava 3,8\% das certificações emitidas.

\subsection{Certificações válidas por setores de atuação}

Após a análise das certificações válidas no período de estudo, realizou-se uma descrição mais detalhada em relação a todas os setores de atuação que apresentavam certificações válidas no período de 2005 a 2012. Tais resultados são explicitados na Tabela 3 abaixo:

Tabela 3- Percentual de certificações válidas por setores de atuação

\begin{tabular}{|c|c|}
\hline Setores de atuação & Percentual de certificações válidas (\%) \\
\hline $\begin{array}{c}\text { Atividades Imobiliárias; Locações e Prestação de } \\
\text { serviços }\end{array}$ & 37,3 \\
\hline Transporte; Armazenagens e Telecomunicação & 33,3 \\
\hline Construção & 16,0 \\
\hline $\begin{array}{c}\text { Atividades de Serviços Sociais Comunitários e } \\
\text { Serviços Pessoais }\end{array}$ & 4,0 \\
\hline $\begin{array}{c}\text { Comércio; Concertos. de veículos auto; bens de } \\
\text { pessoais e domésticos }\end{array}$ & 4,0 \\
\hline Hotéis e Restaurantes & 2,7 \\
\hline Educação & 1,3 \\
\hline Suprimento de Energia Elétrica, gás e água & 1,3 \\
\hline
\end{tabular}

Fonte: Os autores

No período analisado, observou-se um total de 75 certificações válidas, das quais 37,3\% correspondem a atividades imobiliárias; locações e prestação de serviços, seguida por Transporte; armazenagens e telecomunicação com 33,3\% das certificações válidas, sendo que apenas estas duas áreas de atuação correspondem acerca de 70,6\% das certificações válidas no período de estudo. Tal resultado parece corroborar Potoski; Prakash (2005) que ressaltaram que apesar dos custos envolvidos, a certificação pela ISO 14001 pode servir como um dispositivo legitimador das práticas ambientais adotadas pela organização e se configurar como uma ferramenta de redução de custos envolvidos no processo produtivo por meio da redução dos recursos utilizados.

Embora possa haver outras razões envolvidas na adoção das certificações por estes setores, estes setores apresentam grande parte das certificações válidas no período de estudo. Na terceira posição em termos de certificações válidas está a área de atuação da Construção com 16,0\% das 
certificações válidas, área de atuação esta que apresenta quatro vezes mais certificações válidas que as áreas de atuação Atividades de serviços sociais comunitários e serviços pessoais e Comércio; concerto de veículos auto; bens de pessoais e domésticos. Com 2,7\% das certificações válidas encontra-se a área de atuação Hotéis e Restaurantes, seguido de Educação e Suprimento de Energia Elétrica, gás e água. Em relação a estes resultados, observa-se que com exceção de suprimentos de energia elétrica, gás e água, os demais setores estão relacionados a atividades de prestação de serviços.

\subsection{Certificações emitidas por organismos credenciados pelo Inmetro}

Após a análise dos percentuais de certificações emitidas e válidas por área de atuação, realizou-se um levantamento de quais são os cinco maiores organismos certificadores credenciados pelo Inmetro em número de certificações válidas no período de estudo e tal resultado é expresso na Tabela 4:

Tabela 4- Percentual de certificações emitidas por organismos credenciados pelo Inmetro

\begin{tabular}{|c|c|}
\hline Organismos credenciados & Percentual de certificações válidas (\%) \\
\hline BRTÜV - Avaliações da Qualidade Ltda & 78,7 \\
\hline TECPAR - Instituto de Tecnologia do Paraná & 10,1 \\
\hline ABS Quality Evaluations Inc & 4,7 \\
\hline BVQI do Brasil Sociedade Certificadora Ltda & 3,0 \\
\hline $\begin{array}{c}\text { ICQ Brasil - Instituto de Certificação Qualidade } \\
\text { Brasil }\end{array}$ & 1,8 \\
\hline
\end{tabular}

Fonte: Os autores

Analisando-se a tabela acima, observa-se que a maior parte do percentual de certificações válidas foi emitida pela BRTÜV - Avaliações da Qualidade Ltda com cerca de 78,7\% do percentual das certificações válidas. Em segundo lugar, com apenas 10,1\% do percentual das certificações válidas encontra-se a TECPAR- Instituto de Tecnologia do Paraná. Percentual este que é maior do que a soma do percentual de certificações válidas pelos três organismos certificadores que se encontram entre os maiores emissores de certificações válidas, organismos estes que são: ABS Quality Evaluations Inc, BVQI do Brasil Sociedade Certificadora Ltda e ICQ Brasil - Instituto de Certificação Qualidade Brasil.

\section{Conclusão}

O presente estudo que objetivou analisar o processo de certificação pela ISO 14001 no Brasil no período de 2007 a 2012, demonstrou um predomínio dos estados do Sudeste e do Sul no número de certificações válidas e emitidas, sendo que o estado de São Paulo se configurou com o estado com o maior número de certificações válidas e emitidas no período analisado. Isto de certa forma demonstra uma maior percepção do estado de São Paulo acerca dos benefícios associados a implementação da ISO 14001. 
Em relação ao número de certificações emitidas por setores de atuação destacam-se a Atividades Imobiliárias, locações e prestação de serviços e Transporte; armazenagens e telecomunicação apresentam grande parte das certificações válidas no período de 2007 a 2012. Uma maior inserção da norma certificadora ambiental ISO 14001 nestas áreas de atuação pode estar relacionada tanto a uma resposta a pressão exercida por stakeholders sobre estas áreas de atuação quanto à percepção destes setores acerca dos benefícios oriundos da ISO 14001.

Quanto à emissão de certificações, nota-se que apesar da existência de diferentes organismos certificadores credenciados ao Inmentro, as certificações foram emitidas em sua grande maioria pela BRTÜV - Avaliações da Qualidade Ltda.

Devido às limitações deste estudo, já que as empresas certificadas não precisam notificar o Inmetro sobre a certificação recomenda-se para estudos futuros:

- Avaliar as motivações associadas à certificação da ISO 14001 no estado de São Paulo;

- Analisar o motivo de predominância de certificações emitidas pela BRTÜV - Avaliações da Qualidade Ltda em comparação aos demais organismos certificadores;

- Analisar as principais dificuldades enfrentadas pelas empresas certificadas no processo de inserção da ISO 14001;

- Mensurar os fatores incentivadores para implementação da ISO 14001 nas áreas de atuação de Atividades Imobiliárias, locações e prestação de serviços e Transporte; armazenagens e telecomunicação.

\begin{abstract}
Increased environmental concern by government and stakeholders has made the adoption of environmental management systems by organizations has been increasing considerably in recent years. The integration of management systems has been carried out based on regulatory certification such as ISO 14001 currently associated with recognition and wide popularity among businesses. As there is still a lack of studies analyzing thematic ISO 14001 in Brazil, this study aimed to analyze the evolution of the standard ISO 14001:2004 certification from January 2007 to December 2012 based on the information present on the site INMETRO. The research results obtained that the state of São Paulo is the state with the largest number of valid certifications and issued and that the sectors in which real estate activities, rentals and services and transportation, warehousing and telecommunications have much of certifications valid for the period 2007-2012, these certifications issued mostly by the certification BRTÜV - Reviews of Quality Ltd
\end{abstract}

Key-words: ISO 14001, environmental management system; sectors of activity; states.

\title{
Referências
}

ARIMURA, T. H.; DARNALL, N.; KATAYAMA, H. Is ISO 14001 a gateway to more advanced voluntary action? The case of green supply chain management. Journal of Environmental Economics and Management, v. 61, n. 2, p. 170182, 2011. crossref

CAMPOS, LMS et al. Sistema de Gestão Ambiental ISO 14001: Análise Comparativa dos Setores de Atividade entre Regiões e Países. 
CAÑÓN-DE-FRANCIA, J.; GARCÉS-AYERBE, Concepción. ISO 14001 environmental certification: a sign valued by the market?. Environmental and Resource Economics, v. 44, n. 2, p. 245-262, 2009. crossref

DARNALL, N. Why firms mandate ISO 14001 certification. Business \& Society, v. 45, n. 3, p. 354-381, 2006. crossref

DARNALL, N.; HENRIQUES, I.; SADORSKY, P.. Do environmental management systems improve business performance in an international setting?. Journal of International Management, v. 14, n. 4, p. 364-376, 2008. crossref

GAVRONSKI, I.; FERRER, G.; PAIVA, E. L. ISO 14001 certification in Brazil: motivations and benefits. Journal of Cleaner Production, v. 16, n. 1, p. 87-94, 2008. cross ref

GIL, A. C. Como elaborar projetos de pesquisa- 5. Ed; Atlas, São Paulo, 2010.

HART, S. A natural resource-based view of the firm. Academy of Management Review, 1995.

HERAS-SAIZARBITORIA, I.; LANDÍN, G. A.; MOLINA-AZORÍN, J. F. Do drivers matter for the benefits of ISO 14001?. International Journal of Operations \& Production Management, v. 31, n. 2, p. 192-216, 2011. crossref

INMETRO. Instituto Nacional de Metrologia, Normalização e Qualidade Industrial. Relação das empresas certificadoras em atividade. Disponível em: http://www.inmetro.gov.br. Acesso 17/05/2013

IRALDO, F.; TESTA, F.; FREY, M. Is an environmental management system able to influence environmental and competitive performance? The case of the eco-management and audit scheme (EMAS) in the European union. Journal of Cleaner Production, v. 17, n. 16, p. 1444-1452, 2009. crossref

JABBOUR, C. J. C. et al. Environmental management in Brazil: is it a completely competitive priority?. Journal of Cleaner Production, v. 21, n. 1, p. 11-22, 2012. crossref

JABBOUR, C. J. C.; SANTOS, F. C. A.; NAGANO, M. S. Análise do relacionamento entre estágios evolutivos da gestão ambiental e dimensões de recursos humanos: estado da arte e survey em empresas brasileiras. Revista de Administração\&ccdeil; da Universidade de São Paulo, v. 44, n. 4, 2009.

JAFFE, A. B. et al. Environmental regulation and the competitiveness of US manufacturing: what does the evidence tell us?. Journal of Economic literature, v. 33, n. 1, p. 132-163, 1995.

LEÃO, M. T.; SOUBIHIA, D. F.; IZARD, A. L.T.; JABBOUR, C. J. C. Desenvolvimento de produtos com menores impactos ambientais: identificação, classificação e análise da produção científica. XV Seminário em Administração (SEMEAD), outubro 2012.

MARIMON, F.; CASADESÚS, M.; HERAS, I. Certification intensity level of the leading nations in ISO 9000 and ISO 14000 standards. International Journal of Quality \& Reliability Management, v. 27, n. 9, p. 1002-1020, 2010. crossref

MASSOUD, M. A. et al. Drivers, barriers and incentives to implementing environmental management systems in the food industry: A case of Lebanon. Journal of Cleaner Production, v. 18, n. 3, p. 200-209, 2010. crossref

OLIVEIRA, O. J.; SERRA, J. R.; SALGADO, M. H. Does ISO 14001 work in Brazil?. Journal of Cleaner Production, v. 18, n. 18, p. 1797-1806, 2010. crossref

POMBO, F. R.; MAGRINI, A. Panorama de aplicação da norma ISO 14001 no Brasil. Gestão \& Produção, v. 15, n. 1, p. 1-10, 2008. crossref

PRAJOGO, D.; TANG, A. KY; LAI, K. Do firms get what they want from ISO 14001 adoption?-An Australian perspective. Journal of Cleaner Production, 2012. crossref

PRAKASH, A.; POTOSKI, M. Voluntary environmental programs: A comparative perspective. Journal of Policy Analysis and Management, v. 31, n. 1, p. 123-138, 2012. crossref

PEIXE, BCS et al. Certificações de Empresas pelas Normas ISO 14001: Estudo Comparativo e Descritivo da Evolução no Período de 2000 A 2010. 
POMBO, F. R.; MAGRINI, A. Panorama de aplicação da norma ISO 14001 no Brasil. Revista Produção, v. 15, n. 1, p. $1-10,2008$.

PORTER, M. E.; VAN DER LINDE, C. Toward a new conception of the environment-competitiveness relationship. The journal of economic perspectives, v. 9, n. 4, p. 97-118, 1995. cross ref

POTOSKI, M.; PRAKASH, A. Green clubs and voluntary governance: ISO 14001 and firms' regulatory compliance. American Journal of Political Science, v. 49, n. 2, p. 235-248, 2005. crossref

SEIFFERT, M.E.B ISO 14001 Sistemas de Gestão Ambiental- Implantação objetiva e econômica ,4 Ed., Editora Atlas, São Paulo,2011;

SHRIVASTAVA, P. Environmental technologies and competitive advantage. Strategic management journal, v. 16, n. S1, p. 183-200, 1995. crossref

TRUNG, D. N.; KUMAR, S. Resource use and waste management in Vietnam hotel industry. Journal of cleaner production, v. 13, n. 2, p. 109-116, 2005. crossref

QI, G. Y. et al. Diffusion of ISO 14001 environmental management systems in China: rethinking on stakeholders' roles. Journal of Cleaner Production, v. 19, n. 11, p. 1250-1256, 2011. crossref

WALLEY, N.; WHITEHEAD, B. It's not easy being green. Harvard Business Review, 72, 46-52, 1994.

ZIEGLER, A.; SEIJAS NOGAREDA, J. Environmental management systems and technological environmental innovations: Exploring the causal relationship. Research Policy, v. 38, n. 5, p. 885-893, 2009. crossref

\section{Dados dos autores}

Nome completo: Leonardo de Lima Moura

Filiação institucional: Instituto Brasileiro de Mercado de Capitais - IBMEC - RJ.

Departamento: Programa de Pós-Graduação em Logística.

Função ou cargo ocupado: Aluno do Programa de Pós-Graduação em Logística.

Endereço completo para correspondência (bairro, cidade, estado, país e CEP): Rua João Barbalho, 127, casa 11, Quintino, Rio de Janeiro, RJ.

Telefones para contato: (55) 2172837156.

e-mail: mouraventura@uol.com.br

Nome completo: Ronaldo Ferreira da Silva

Filiação institucional: Universidade Federal Fluminense - UFF - RJ.

Departamento: Assistência Farmacêutica.

Função ou cargo: Mestre em Sistemas de Gestão - UFF.

Endereço completo para correspondência (bairro, cidade, estado, país e CEP): Rua Marquês do

Paraná 282 Centro, Niterói, RJ - CEP 24030-190 - Telefones: (21) 2629-9444 e 2629-9445.

e-mail: ronaldorubanouff@gmail.com

Submetido em: 25/07/2013

Aceito em: 31/01/2014 